\title{
Necesidades educativas especiales: una mejora mediante innovación educativa
}

\author{
Juan José Maldonado Briegas, Sergio González Ballester, Dolores Vera González, Florencio Vicente Castro \\ Facultad Educación (UNEX)
}

\begin{abstract}
Resumen
Según el último informe de la Comisión Europea sobre educación, discapacidad y necesidades especiales (NESSE, 2012) "Alrededor de 15 millones de niños de la Unión Europea tienen necesidades educativas especiales (NEE)". Como consecuencia, muchos de ellos cursan estudios en centros especiales y/o no reciben el apoyo adecuado por el sistema educativo. Con todo ello, planteamos un estudio para valorar los beneficios de la aplicación de la robótica educativa en el aprendizaje de niños con NEE. Los resultados mostraron, que la innovación educativa basada en la robótica ayuda a crear ambientes interdisciplinarios y promueve la inclusión y el trabajo colaborativo.

Palabras clave: robótica, aprendizaje, inclusión, TDAH.
\end{abstract}

\begin{abstract}
According to the latest report of the European Commission on education, disability and special needs (NESSE, 2012) "About 15 million children in the European Union have special educational needs (SEN)". As a result, many of them attend special education centers and / or do not receive adequate support from the education system. With this, we propose a study to evaluate the benefits of the application of educational robotics in the learning of children with SEN. The results showed that robotics-based educational innovation helps create interdisciplinary environments and promotes inclusion and collaborative work.

Key words: robotics, learning, inclusion, ADHD.
\end{abstract}

\section{Introducción}

A finales del siglo XX las autoridades mundiales, en la Conferencia Mundial sobre Educación para Todos: Satisfacción de las Necesidades Básicas de Aprendizaje (Jomtien, Tailandia, 1990), y la Cumbre Mundial en favor de la Infancia (Nueva York, 1990), aprobaron el objetivo de la Educación para Todos antes del Año 2000.

Se iniciaron, pues, los primeros pasos para hacer llegar el conocimiento y las aptitudes a cualquier niño que presentase cualquier tipo de dificultad, independientemente del origen del mismo. De esta forma los niños con necesidades educativas especiales (NEE) se establecieron como un elemento fundamental en la defensa de la integración en la sociedad y como un motor de cambio del sistema educativo al fomentar la apertura de escuelas a todos los niños, erigiéndose los centros educativos como medios pedagógicos agradables y estimulantes para la adquisición de conocimientos.

Desde esa fecha hasta la actualidad el sistema educativo ha evolucionado de tal forma que ha exigido cambios en la confección de los centros de educación para poder cumplir con la atención específica de los alumnos con necesidades educativas especiales (NEE). Esto es debido a que los centros educativos con esta tipología de alumnos demanda nuevos recursos de personal, personal cualificado, recursos didácticos y nuevos materiales.

Estas demandas de los centros educativos vendrán definidas por la clase de alumnos que asista al centro, ya que tendrán unos requerimientos educativos específicos en función de su necesidad educativa, y que son necesarios para su desarrollo personal y social; y los instrumentos o recursos que vayan a ser utilizados conforme a lo establecido por el profesorado del centro.

$\mathrm{Si}$ bien es sabido que el principal instrumento didáctico con que cuenta la enseñanza actual, es el libro de texto, éste no está concebido como recurso de aprendizaje que facilite la adquisición de competencias de aquel alumnado que experimenta dificultades al enfrentarse al currículum escolar. Su carácter graduado, disciplinar, su arcaica metodología memorística o su concepción del trabajo escolar como tarea individual llega a hacerlo particularmente ineficaz.

Es por ello que en este artículo pretendemos recopilar distintos tipos planteamientos educativos con base en la robótica que puedan servir como reseña para elaborar propuestas de intervención metodológicas innovadoras de aprendizaje.

\section{Objetivos}

El objetivo de esta revisión es, por tanto, recopilar distintos tipos de enfoques educativos que tengan como denominador común la robótica y que puedan servir como punto de referencia para elaborar una propuesta de intervención metodológica de aprendizaje. El empleo de la robótica como eje central del aprendizaje en niños con NEE y concretamente en alumnos con TDAH, ayudaría posiblemente a mejorar su control, su comportamiento y conducta durante el proceso de adquisición de competencias. 


\section{Método}

La investigación realizada se ha desarrollado bajo dos ópticas concordantes: a) por un lado, el aprendizaje de la robótica y, b) por otro, el aprendizaje usando la robótica. Con el objetivo de que los niños aprendan a definir de forma conceptual robots bajo un proceso creativo que le permita resolver situaciones o desarrollar actuaciones bajo un determinado contexto o necesidad en diferentes áreas del conocimiento, y de igual forma se instruyan en la construcción física de robots en relación con el contexto o situación a resolver, realizando operaciones de programación, sensorización, procesos de montajes de forma dinámica, todo ello bajo el paraguas del aprendizaje colaborativo.

Se realizó una búsqueda bibliográfica electrónica por uno de los revisores (S.G.B.) a través de diferentes buscadores Scielo, Dialnet, Google Academic, WoldWideScience y SpringerLink. No se aplicaron restricciones de idiomas siendo la búsqueda limitada a los últimos diez años (Enero 2007 a Abril 2017). Debido a las características de los estudios analizados, sólo pudo realizarse un análisis cualitativo de los resultados obtenidos. La pregunta clave que guio esta revisión fue: ¿existen beneficios del uso de la robótica educativa como herramienta para el aprendizaje de niños con NEE y concretamente con (TDAH)?

\section{Estrategia de búsqueda}

La estrategia de búsqueda se fundamentó en una serie de términos controlados y palabras clave, definidos por los siguientes tesaruos: robótica, educación, enseñanza y TDAH. Los términos de búsquedas empleados, identificados en el título o abstract, fueron: "Robótica" AND "educación” OR "enseñanza" OR "programa educativo" OR "programa de intervención" AND “ necesidades educativas especiales" OR “ TDAH” OR “ Trastorno con déficit de atención e hiperactividad" OR "NEE". Los buscadores Scielo, Dialnet, SpingerLink y WorldWideScience sólo mostraban resultados si sólo se establecían dos términos de búsqueda, por lo que se decidió llevar a cabo combinaciones de tesauros de la siguiente forma: "Robótica" AND "educación" OR "enseñanza"; "Robótica" AND "programa educativo" OR "programa de intervención"; "Robótica" AND "necesidades educativas especiales" OR "TDAH" OR "Trastorno con déficit de atención e hiperactividad" OR “ NEE"; "Educación" OR "Enseñanza" AND "TDAH" OR "NEE"; "Intervención" AND "TDAH" OR “ Trastorno con déficit de atención e hiperactividad" OR "NEE". Las combinaciones realizadas se realizaron tanto en lengua española como en lengua inglesa.

\section{Criterio de elegibilidad}

Los artículos incluidos en esta revisión sistemática fueron incorporados si cumplían con los siguientes criterios de inclusión: estudios de programas de intervención con robótica en el ámbito escolar, estudios innovadores con robótica, estudios de aplicación de la robótica en el aprendizaje y dirigidos a alumnos con NEE y a su vez centrados en TDAH.

Por el contrario, se excluyeron los estudios que no cumplían los criterios anteriormente citados.

\section{Proceso de cribado y extracción de datos}

El proceso de cribado fue realizado por cuatro revisores (F.V.C.;J.J.M.B.;S.G.B.;D.V.G.). Los títulos y resúmenes fueron analizados en primer lugar.

El segundo paso consistió en la selección de determinados artículos relacionados con la pregunta clave. Todos los artículos seleccionados fueron detalladamente analizados conforme a los criterios de elegibilidad para la posterior extracción de datos. De cada estudio incluido en la revisión se extrajo la siguiente información: primer autor, año de publicación, diseño del estudio, número de sujetos, tipo de actuación, beneficios obtenidos de la robótica educativa.

\section{Resultados}

Búsqueda y análisis de Literatura

La estrategia de búsqueda inicial arrojó un total de 269 documentos, entre ellos 4 libros, 2 ensayos, 37 Trabajos Fin de Grado, 9 Trabajos Fin de Máster, 201 Artículos y 16 Tesis de las bases de datos de Scielo, Dialnet, Google Academics, WorldWideSience y SpringerLink. 44 documentos obtenidos de citas relacionadas en Scielo, 55 documentos obtenidos de Dialnet, 144 documentos obtenidos de Google Academics, 20 documentos procedentes de WorldWideSience y 6 documentos de SpringerLink.. En el primer procedimiento llevado a cabo se revisaron los títulos y resúmenes de los artículos obtenidos en la búsqueda de estrategias para la eliminación de documentos irrelevantes (192 artículos). En el segundo paso, se analizaron los documentos relacionados con la pregunta clave (77 documentos).

Posteriormente, se realizó una lectura exhaustiva y detallada del texto completo de cada uno de los artículos seleccionados (47 documentos). Finalmente, sólo 3 documentos, todos ellos artículos, fueron incluidos para la extracción de datos finales [Figura 1]. Debido a la tipología de la investigación y heterogeneidad de los estudios analizados se realizó una síntesis cualitativa de los datos. 


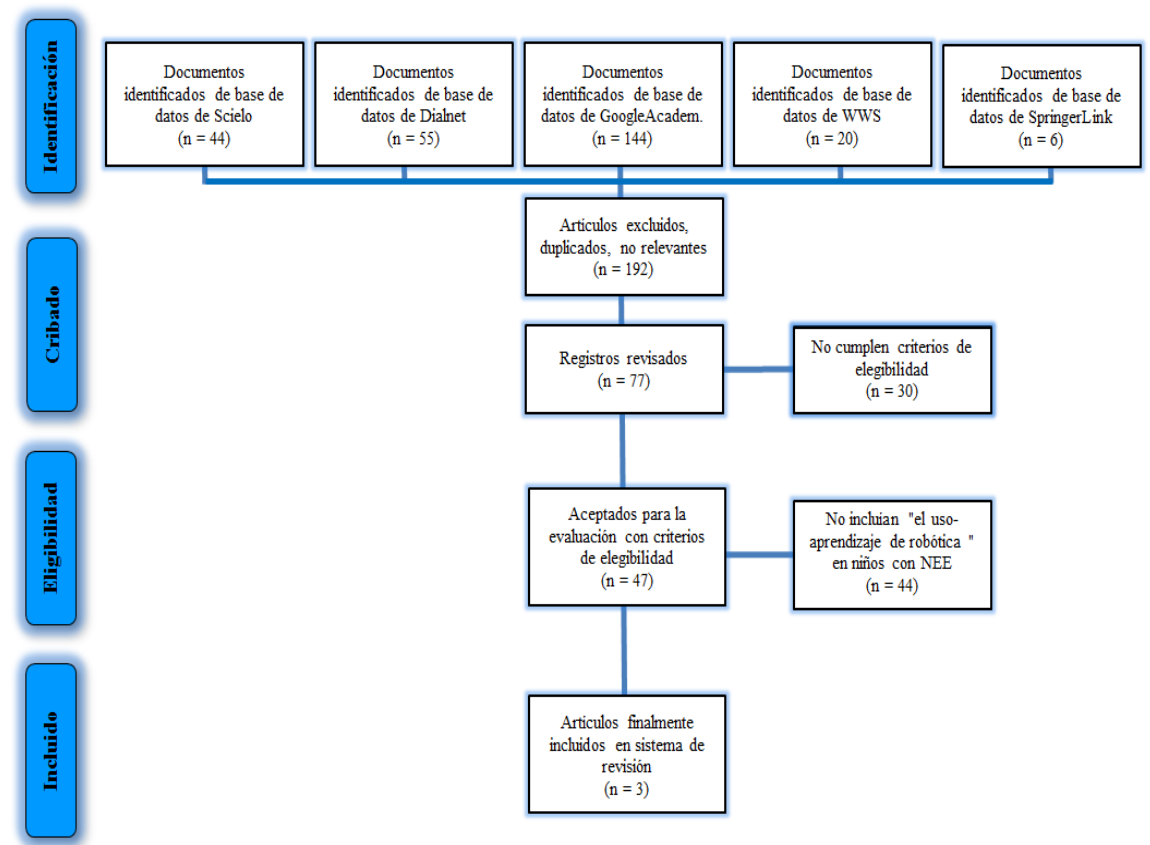

Figura 1. Diagrama de flujo (proceso de selección y selección) utilizado para los artículos incluidos en esta revisión

Características de los estudios

Las características de los estudios incluidos en la presente revisión se muestran en las Tablas 1 y 2 . Se incluyeron estudios referentes a la aplicación de la robótica como herramienta educativa en el aprendizaje de niños con NEE, y los enfocados principalmente a niños con TDAH. Para ello se seleccionaron estudios referentes a programas de intervención desarrollados en el ámbito escolar, así como estudios relacionados con intervenciones innovadoras educativas en el que tuvieran como eje central la robótica. Se excluyeron dentro de esta revisión aquellos estudios en los que se utilizaban robots asistenciales para el aprendizaje en niños con NEE por no cumplir la directriz de "tener que aprender con el uso de la robótica", realizando un aprendizaje dinámico.

Estudios relacionados con la aplicación de la robótica en el aprendizaje de niños con NEE

De los cuarenta y siete documentos revisados sólo tres de artículos de los elegidos estudiaron el potencial inclusivo de la robótica educativa (RE) en alumnos con NEE. Sólo uno de ellos [1] hacía referencias a niños con TDAH.

El primero de los estudios [1] correspondía a un taller de formación orientado a dos terapeutas que pusieron en práctica la aplicación de la robótica con tres alumnos con NEE (uno de ellos presentaba DAE, otro presentaba TDAH y el tercer alumno presentaba DPA). En el estudio se comparaba el potencial pedagógico y terapéutico de la robótica tangible y virtual.

En este estudio la utilización de la robótica tenía el fin de capacitar a los participantes para montar, programar e interactuar con el conjunto educativo de Lego® y el simulador de robótica virtual RoboMind®, con el objeto de fomentar el aprendizaje, la rehabilitación motora y el desarrollo del razonamiento lógico y deductivo y del pensamiento computacional.

Como recurso se utilizó el Kit educativo do Lego® Mindstorms ${ }^{\circledR}$ NXT® (herramienta robótica tangible), así como RoboMind ${ }^{\circledR}$ (herramienta robótica virtual).

Los participantes disfrutaron de las actividades y afirmaron el gusto de realizar una actividad diferente en la terapia.

Los beneficios que destacaron que aporta la RE fueron: el desarrollo de varias habilidades $y$ competencias de los niños, como la creatividad, y que la terapia basada en la robótica (tangible o virtual), podría ser "una intervención interesante para el desarrollo de niños con trastornos del espectro del autismo (PEA), niños con TDAH, niños con dificultades de aprendizaje específicas y niños con capacidades cognitivas superiores".

El segundo estudio [2] revisado presenta una estrategia de inclusión y consolidación del conocimiento basada en el aprendizaje lúdico mediante proyectos de robótica educativa (RE).

La actividad robótica, basada en el aprendizaje a través de juegos y proyectos, consistió en programar e interactuar con un conjunto de Lego ${ }^{\circledR}$ Mindstorms ${ }^{\circledR}$ NXT® con el objetivo de promover la inclusión, interacción y aprendizaje de nuevos contenidos en varias materias, incluyendo lengua portuguesa, matemáticas, programación y educación cívica.

En el estudio participaron tres niños con edades comprendidas entre 14 y 15 años de edad y a los que se les había diagnosticado dificultad de aprendizaje Learning Disabilities (LD)

Se utilizó como material didáctico el kit de aprendizaje de Lego® Mindstorms ${ }^{\circledR}$ NXT.

Los resultados mostraron que la $\mathrm{RE}$ es una herramienta de gran potencial académico que tiende a 
Tabla 1: Resumen de principales características de estudios revisados

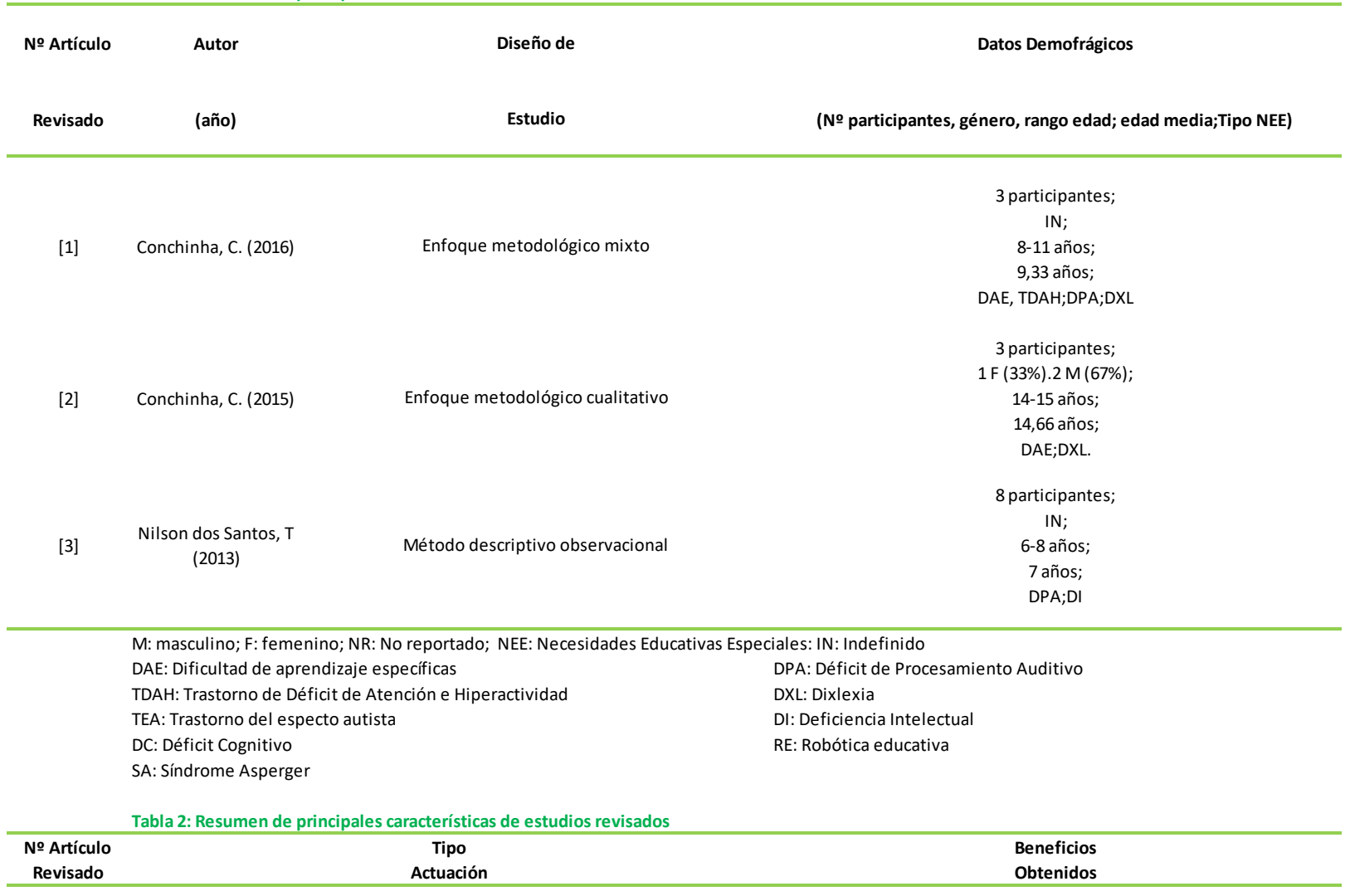

[1]

Taller formación para capacitación de profesionales para uso robótica educativa (RE) en contexto inclusivo aplicada a NEE. Caso práctico en 3 niños con NEE.
La RE favorece el desarrollo de creatividad, habilidades y competencias. La terapia basada en la RE puede ser una intervención interesante para el desarrollo de niños con (PEA), TDAH, niños con dificultades de aprendizaje específicas y niños con capacidades cognitivas superiores. El aprendizaje con RE mostró niños más atentos y concentrados.
[2]

Proyecto para programar e interactuar con un conjunto educativo de Lego ${ }^{\circledR}$ Mindstorms ${ }^{\circledR} \mathrm{NXT}^{\circledast}$ con el fin de promover la inclusión, interacción y aprendiz matemáticas, programación y educación cívica.
La RE una herramienta con potencial académico e inclusivo cuando se utilizó con estudiantes con dificultades de aprendizaje.

Las actividades de RE colaborativas y lúdicas basadas en proyectos tienden a motivar a los estudiantes.

Realizacion de actividad que involucraba la robótica educativa en la disciplina de Los niños sin discapacidad demostraron más interés en descubrir el matemáticas para niños con y sin NEE. Análisis comparativo de sus reacciones, el problema que se resolvería que los niños con discapacidad. Sin tiempo de ejecución de las actividades, el interés, dedicación, atención, e embargo, todas participaron en la actividad, interactuando y interacción. cuestionando.

M: masculino; F: femenino; NR: No reportado; NEE: Necesidades Educativas Especiales: IN: Indefinido

DAE: Dificultad de aprendizaje específicas

TDAH: Trastorno de Déficit de Atención e Hiperactividad

TEA: Trastorno del especto autista

DPA: Déficit de Procesamiento Auditivo

DC: Déficit Cognitivo

SA: Síndrome Asperger

DXL: Dixlexia

DI: Deficiencia Intelectual

RE: Robótica educativa

motivar al alumno al sentirse éste desafiado a completar las actividades que se le proponen. Así mismo es un recurso que favorece las relaciones entre los participantes, promueve la inclusión e igualdad, en alumnos con LD, y favorece la adquisición de conocimientos. Por otra parte se contempla como un instrumento útil para resolver problemas cotidianos de los estudiantes al favorecer el uso del lenguaje y las relaciones interpersonales.

En el estudio [3] se analizaba la aplicación de la RE en la disciplina de matemáticas para niños con enseñanza especial, niños con NEE, y niños con enseñanza regular, con el objetivo de establecer un comparativo del comportamiento de estos niños después del análisis de sus reacciones y del tiempo de ejecución de las actividades.

La actividad tenía el objetivo de estimular en los niños el entendimiento de las dos principales operaciones matemáticas - Suma y sustracción.

En la investigación se realizaron análisis de sus reacciones, interés, dedicación, atención, interacción, entre otros.

El recurso utilizado en este estudio fue el Lego Mindstorms NXT 2.0.

Los resultados identificaron que los niños sin discapacidad demostraron más interés en descubrir el 
problema que se debía resolver que los niños con discapacidad. Todos los niños participaron en la actividad, interactuando y cuestionando.

\section{Discusión}

La robótica educativa (RE) percibida hoy en día como un medio canalizador del aprendizaje, se sustenta en el principio piagetiano de que si no hay intervención del estudiante en la construcción del objeto de conocimiento no existe aprendizaje alguno (Ruíz, 2007).

Esta concepción de la robótica ha permitido, de forma paulatina, su integración en el proceso de enseñanza-aprendizaje al conseguir estimular el potencial creativo y expresivo de los usuarios a través del desarrollo de proyectos, resolución de problemas (Wagner, 1998) favoreciendo razonamiento crítico (Sendag et al., 2009) y el trabajo en equipo.

El objetivo de este artículo ha sido recopilar distintos tipos de enfoques educativos que tengan como denominador común la robótica y que puedan servir como punto de referencia para elaborar una propuesta de intervención metodológica de aprendizaje innovadora en el aula, y que ayuden a mejorar el control, el comportamiento y la conducta de niños con NEE, y concretamente los diagnosticados con síndrome TDAH.

Sólo tres artículos fueron finalmente incluidos en esta revisión de acuerdo con nuestra estrategia de revisión.

Entre ellos se constata que la RE establece una tendencia positiva al desarrollo del lenguaje, contemplándose como un instrumento útil para resolver problemas cotidianos entre los estudiantes al favorecer ese uso del lenguaje y las relaciones interpersonales, a la vez que fomenta el aprendizaje de nuevos vocablos en otros idiomas.

Hecho que confirman otros autores que sostienen que la robótica invita a la resolución de problemas (Barnes, 2002; Mauch, 2001; Nourbakhsh et al., 2005; Robinson, 2005; Rogers y Portsmore, 2004) y promueve el aprendizaje cooperativo (Beer et al., 1999; Nourbakhsh et al., 2005).

Por otro lado la RE evidenció, en varios de los estudios revisados, que conseguía incrementar el razonamiento lógico-matemático, a la vez que lograba aumentar la motivación del alumnado sobre la materia propuesta facilitando el aprendizaje materias como las matemáticas, informática y tecnología; demostraba, de igual forma, que los niños adquieren, de forma práctica, competencias transversales como el trabajo en equipo, búsqueda de información y resolución de problemas.

Estos resultados son concordantes con otras investigaciones realizadas en el contexto educacional que mostraron que la actividad robótica provoca un efecto positivo de motivación en el aula (Barker y Ansorge, 2007; Gura, 2007; Nourbakhsh et al., 2005), así como una efectividad potencial para impactar positivamente en el aprendizaje (Fagin y Merkle, 2003).
Los beneficios destacados fueron el desarrollo de varias habilidades y competencias de los niños, así como la creatividad, y que la terapia basada en la robótica podría ser una intervención interesante para el desarrollo de niños diferentes tipos de trastornos.

A su vez la RE favoreció las relaciones entre los participantes, promoviendo la inclusión e igualdad, y de forma especial en pacientes con LD (Dificultad en el aprendizaje), a la vez que impulsó la adquisición de conocimientos de diversas áreas temáticas.

\section{Conclusiones}

Tras la revisión realizada concluimos que la robótica educativa constituye una herramienta útil en el desarrollo de múltiples facetas de los alumnos, sobre todo en aquellos con NEE, alteraciones del comportamiento, déficit de atención etc., ayudándoles a adquirir nuevas competencias y mejorar sus habilidades interpersonales, impulsar su creatividad, aumentar su concentración, mejorar sus resultados académicos y por ende su autoestima.

Seguramente con los nuevos avances tecnológicos y con las innovadoras propuestas educativas, en un futuro próximo, la robótica podrá establecerse como una herramienta cotidiana de aprendizaje en las aulas permitiendo adaptarse a las singularidades $y$ necesidades educativas de cada alumno de forma que consiga una mejor adaptación a los tiempos de aprendizaje de éstos, así como una mayor motivación, involucración y adquisición de conceptos con gran implicación por su parte.

\section{Limitaciones del estudio}

Los autores de este documento reconocen las limitaciones inherentes a esta revisión. El estudio se limita a cinco bases de datos (Scielo, Dialnet, Google Academic, WoldWideScience y SpringerLink) y sólo quince artículos fueron incluidos en el estudio final de acuerdo con nuestra estrategia de búsqueda. El escaso número de estudios encontrados relacionados con la aplicación de la robótica en el aprendizaje de niños con NEE, la variedad de diseño de estudios, las limitaciones encontradas en muchas de las investigaciones, junto con el hecho de que existe ausencia de cierta información referente a los grupos de participantes sobres los que se describen los estudios hacen que los estudios sean bastante heterogéneos para obtener cualquier tipo de resultados cuantitativos. Por lo tanto, las conclusiones obtenidas deben tomarse con cautela.

Tal vez, los estudios futuros definan de una forma más concreta y sistemática programas de intervención, o propuestas innovadoras, con la robótica como recurso didáctico central, y dirigidos a cualquier tipo de alumno incluidos los pacientes con NEE de forma que se pueda proporcionar información más confiable para su aplicación en la rutina diaria del aula.

\section{Referencias}

Astolfo, D., Ferrari, M., \& Ferrari, G. (2007). Building Robots with LEGO Mindstorms NXT. Ed. Syngress. 
Bagnall, B. (2007). "Maximum LEGO NXT: Building Robots with Java Brains". Variant Press.

Batzogiannis,I.;Hatzikraniotis,E. (2015). Can innovative learning applications influence the students' attitudes towards science: The case of educational robotics. Proceedings of the 1th D4|Learning international Conference Innovations in Digital Learning for Inclusion.(D4Learning, 2015). (Open access edition ed.) Aalborg Universitetsforlag, pp. 175-179.

Benito Rodríguez, A; Ramírez López, L.J.; Fernández,W. (2017). Metodologías Activas para Alcanzar el Comprender. Formación Universitaria. Vol. 10(1), 79-88 (2017).

Berlin C, Jose Uc, González, C.; García, M.;Escalante, M.; Montañez,T. (2015). Análisis Comparativo de dos Formas de Enseñar Matemáticas Básicas: Robots LEGO NXT y Animación con Scratch.

Castro Rojas,M.D.; Acuña Zúñiga, A.L. (2012). Propuesta comunitaria con robótica educativa: valoración y resultados de aprendizaje. Revista Teoría de la Educación: Educación y Cultura en la Sociedad de la Información. 13(2), 91-118. http://campus.usal.es/ revistas_trabajo/index.php/re vistatesi/article/view/9001/9246

Conchinha, C; Honório Rodrigues, A.C.; Pires Nogueira; A; Correia de Freitas,J. (2016). A robótica como ferramenta coadjuvante na formação $e$ reabilitação de crianças com NEE. Libro de Actas. Conferencia Ibérica en Innovación en la Educación $\begin{array}{lll}\text { con TIC. } & \text { ieTIC }\end{array}$ http://www.conferencias.ipb.pt/index.php/ieTIC201 6

Conchinha, C; Osório,P.; Correia de Freitas,J. (2015). Playful learning: Educational Robotics applied to Students with Learning Disabilities. SIIE'15: XVII International Symposium on Computers in Education.

Hepp,P.; Merino, M.E.; Barriga,M.V; Huircapán,A. (2013). Tecnología robótica en contextos escolares vulnerables con estudiantes de la etnia Mapuche. Estudios Pedagógicos XXXIX, Número Especial 1: 75-84, 2013.

Huskens,B.; Palmen,A. ; Van der Werff,M.; Lourens,T.;Barakova,E. (2015). Improving Collaborative Play Between Children with Autism Spectrum Disorders and Their Siblings: The Effectiveness of a Robot-Mediated Intervention Based on Lego ${ }^{\circledR}$ Therapy. Autism Dev Disord . 45:3746-3755. Springer Science+Business Media New York 2014.

Kelly, J. (2006). LEGO Mindstorms NXT: The Mayan Adventure. Ed. Apress.

García Y. y Reyes, D. (2012). Robótica Educativa y su potencial mediador en el desarrollo de las competencias asociadas a la alfabetización científica. Educación y Tecnología, 2, 42-55.

Kelly, J., \& Daudelin, J. (2008). "FIRST LEGO League: The Unofficial Guide”. No Starch Press.

Marchesi, A., Martín, E., Casas, E., Ibáñez, A., Monguillot, I., Rivière, V., et al. (2003). Tecnología y aprendizaje. Investigación sobre el impacto del ordenador en el aula. Madrid: SM.

Márquez D.,J.E.; Ruiz F.;J.H. (2014). Robótica educativa aplicada a la enseñanza básica secundaria. Revista DIM / Año 10 - No 30 diciembre 2014

Martínez, F.J. (2009) "Las Tecnologías de la Información y la Comunicación (TIC) y las Competencias Básicas en Educación”. Espiral. Cuadernos del Profesorado, 2, 5, 15-26.

Nesse (2012). Education and Disability/Special Needs - policies and practices in education, training and employment for students with disabilities and special educational needs in the EU. Disponible en: http://www.nesse.fr/nesse/activities/reports/activities /reports/disability-special-needs-1

Nilson dos Santos, T; Pozzebon,E;Bolan Frigo,L. (2013). Robótica Aplicada à Educação Especial. ICBL2013 - International Conference on Interactive Computer aided Blended Learning.

Ocaña Rebollo, G. (2012). Robótica como asignatura en enseñanza secundaria. Resultados de una experiencia educativa. Espiral. Cuadernos del Profesorado, 5(10), 56-64. Disponible en: http://www.cepcuevasolula.es/espiral.

Ocaña Rebollo, G. (2010a). Robótica en el Aula. Una experiencia tecnológica para el desarrollo de Competencias Básicas. Paradigma Digital. Revista de Divulgación Educativa,12, 160-173.

Papert, S. (1992). The Children's Machine. Basic Books.

Papert, S. (1980). Mindstorms: Children, Computers, and Powerful Ideas. Basic Books.

Papert, S. (1986). Construcionism: a new opportunity for elementary science education; a proposal to the National Science Foundation. MIT, Media Lab.

Pereira Junio, C.A. (2014). Robótica educacional aplicada ao ensino de química: colaboração $e$ aprendizagem. Tesis.2014.

Petry, P. P. (1996). Processos cognitivos de professores num ambiente construtivista de robótica educacional. 174p. Dissertação (Mestrado em Psicologia) - Universidade Federal do Rio Grande do Sul, Porto Alegre.

Rocha, R. (2006). Utilização da Robótica Pedagógica no Processo de Ensino-Aprendizagem de Programação de Computadores. 115p. Dissertação (Mestrado em Educação Tecnológica) - Centro Federal de Educação Tecn. de Minas Gerais, Belo Horizonte.

Santos, C. F. (2005). Um Estudo sobre Robótica Educacional usando Lego Mindstorm. 166p. Dissertação (Mestrado em Informática) Universidade Federal do Espírito Santo, Vitória.

Santos, F. F. (2004). A robótica educacional como ambiente para a produção de significados no ensino médio. 125p. Dissertação (Mestrado em Educação) Universidade Estácio de Sá, Rio de Janeiro.

Sheehy;K; Ferguson,R. (2008). Educational inclusion and new technologies. In: Scott, Thomas B. and Livingston, James I. eds. Leading-Edge Educational 
Technology. New York: Nova Science Publishers, pp. 159-176.

Silva Andrade, F.J. (2011). Uma Metodologia educacional no estudo de Funções de $7^{\circ}$ ano. Universidade de Madeira.

Vega-Moreno, D., Cufíé, X., Rueda, Mª J, \& Llinás, D. (2016). Integración de robótica educativa de bajo coste en el ámbito de la educación secundaria para fomentar el aprendizaje por proyectos. International Journal of Educational Research and Innovation (IJERI), 6, 162-175 